\title{
Erratum
}

S. Fischler · T. Rivoal

\section{Erratum to: Rational approximation to values of $G$-functions, and their expansions in integer bases}

Published online: 3 January 2018

\section{Erratum to: manuscripta math. DOI 10.1007/s00229-017-0933-8}

In this note we correct a mistake in the proof of the main result of [1], pointed out to us by Dimitri Le Meur.

At the beginning of $\S 2.3$ of [1] appears the inequality

$$
H\left(A_{1} \cdots A_{n}\right) \leq\left(\begin{array}{c}
d_{1}+\cdots+d_{n} \\
n-1
\end{array}\right) H\left(A_{1}\right) \cdots H\left(A_{n}\right)
$$

for polynomials $A_{j}$ of degree less than or equal to $d_{j}$. Actually, this upper bound is false in general, as the example $A_{j}=X+1$ for any $j$ shows. However, this mistake has no consequence on the rest of [1] because this inequality was used only to prove that

$$
H\left(Q_{k}\right) \leq 2^{2 q+(d-1) k+1} H(\mathcal{D})^{k} H(Q),
$$

and we shall prove now that Eq. (1) holds.

Indeed, for any $A, B \in \mathbb{C}[X]$, we have

$$
H(A B) \leq \min (1+\operatorname{deg} A, 1+\operatorname{deg} B) H(A) H(B)
$$

so that

$$
H\left(\mathcal{D}^{k}\right) \leq(1+\operatorname{deg} \mathcal{D})^{k-1} H(\mathcal{D})^{k} \leq(d+1)^{k-1} H(\mathcal{D})^{k}
$$

and

$$
H\left(Q_{k}\right) \leq c_{k} H(\mathcal{D})^{k} H(Q)
$$

for any $k \geq 0$, where $c_{k}=0$ if $k>q$ and, if $k \leq q$,

$$
\begin{aligned}
c_{k} & =\min \left(1+k \operatorname{deg} \mathcal{D}, 1+\operatorname{deg} Q^{(k)}\right)(d+1)^{k-1} 2^{q} \\
& \leq(q-k+1)(d+1)^{k-1} 2^{q} \\
& \leq 2^{q-k}(d+1)^{k} 2^{q} \\
& \leq 2^{2 q}\left(\frac{d+1}{2}\right)^{k} \\
& \leq 2^{2 q+(d-1) k}
\end{aligned}
$$

The original article can be found online at https://doi.org/10.1007/s00229-017-0933-8.

S. Fischler $(\varangle)$ : Laboratoire de Mathématiques d'Orsay, CNRS, Univ. Paris-Sud, Université Paris-Saclay, 91405 Orsay, France. e-mail: stephane.fischler@math.u-psud.fr

T. Rivoal: Institut Fourier, CNRS et Université Grenoble Alpes, CS 40700, 38058 Grenoble Cedex 9, France. 
where the last inequality comes from the fact that $\frac{d+1}{2} \leq 2^{d-1}$ for any positive integer $d$.

\section{Reference}

[1] Fischler, S., Rivoal, T.: Rational approximation to values of $G$-functions, and their expansions in integer bases. Manuscripta math. (2017). https://doi.org/10.1007/s00229-0170933-8 